\title{
Hsa-miR-137, hsa-miR-520e and hsa-miR-590-3p perform crucial roles in Lynch syndrome
}

\author{
CHANGYU ZHOU ${ }^{1}$, JIAYU LI ${ }^{2}$, JIARUI LI ${ }^{3}$, YINGCHUN WAN ${ }^{4}$, TAO LI ${ }^{5}$, \\ PIYONG MA ${ }^{6}$, YINGJIAN WANG ${ }^{7}$ and HAIYAN SANG ${ }^{2}$ \\ ${ }^{1}$ Digest Department; ${ }^{2}$ Department of Cardiology, China-Japan Union Hospital of Jilin University, Changchun, Jilin 130033; \\ ${ }^{3}$ Pharmacy Department, Tumor Hospital of Jilin, Changchun, Jilin 130012; \\ Departments of ${ }^{4}$ Endocrinology, ${ }^{5}$ Anesthesiology, ${ }^{6}$ Emergency Medicine and ${ }^{7}$ Gynaecology, \\ China-Japan Union Hospital of Jilin University, Changchun, Jilin 130033, P.R. China \\ Received February 5, 2015; Accepted May 17, 2016
}

DOI: $10.3892 / \mathrm{ol} .2016 .4816$

\begin{abstract}
The aim of the present study was to identify the differentially expressed microRNAs (DEMs) between Lynch syndrome (LS) and the normal colonic (N-C) control samples, predict the target genes (TGs) and analyze the potential functions of the DEMs and TGs. The miRNA expression dataset GSE30454, which included data of 13 LS and $20 \mathrm{~N}-\mathrm{C}$ tissue samples, was downloaded from the Gene Expression Omnibus. The classical $t$-test in Linear Models for Microarray Data package was used for DEM identification. TG prediction was performed using 5 databases. The regulatory network of the DEMs and their TGs was constructed using Cytoscape. Functional and pathway enrichment analysis was performed. The transcription factors (TFs), tumor-associated genes (TAG) and tumor suppressor genes (TSGs) were then identified. Three key DEMs hsa-miR-137, hsa-miR-520e, and hsa-miR-590-3p were identified.Hsa-miR-520e and hsa-miR-137 had 4 common TGs, including SNF related kinase, metal-regulatory transcription factor 1 (MTF1), round spermatid basic protein 1 and YTH N6-methyladenosine RNA binding protein 3; hsa-miR-590-3p and hsa-miR-137 had 14 common TGs, including NCK adaptor protein 1 (NCK1), EPH receptor A7, and stress-associated endoplasmic reticulum protein 1; hsa-miR-590-3p and hsa-miR-520e had 12 common TGs, including Krüppel-like factor $(K L F)$ 13, twinfilin actin binding protein 1, and nuclear factor I B. Through the functional and pathway enrichments analysis, MTF1 was involved in regulation of gene expression and metabolic processes, and sequence-specific DNA binding TF activity. KLF13 was involved in regulation of gene expression and regulation of cellular metabolic processes. NCK1 was
\end{abstract}

Correspondence to: Dr Jiayu Li, Department of Cardiology, China-Japan Union Hospital of Jilin University, 126 Xiantai Street, Changchun, Jilin 130033, P.R. China

E-mail: jiayulily@163.com

Key words: Lynch syndrome, differentially expressed miRNA, target gene, regulatory network enriched in the axon guidance pathway. In addition, the functional and pathway enrichment analysis showed certain TGs, such as hypoxia-inducible factor $1 \alpha$, AKT serine/threonine kinase 2, and rapamycin-insensitive companion of mammalian target of rapamycin, participated in the mTOR signaling pathway. The 3 key DEMs hsa-miR-137, hsa-miR-520e, and hsa-miR-590-3p may have important roles in the process of LS.

\section{Introduction}

Colorectal cancer (CRC) is one of the most common causes of cancer-associated mortality and is the second and third leading cause of cancer-associated mortality in Western countries and the USA, respectively (1,2). In 2014, $\sim 137,000$ individuals in the USA were diagnosed with CRC and $~ 50,000$ patients succumbed to CRC. In addition, more than one-third of all CRC-associated mortalities (29\% in men and $43 \%$ in women) occurred in individuals aged $\geq 80$ years (1). Lynch syndrome (LS), previously termed hereditary non-polyposis colorectal cancer (HNPCC), accounts for 3\% of all CRCs and is caused by a germline mutation in one of the mismatch repair (MMR) genes mutL homolog 1 (MLH1), mutS homolog $(M S H) 2$, MSH6 and PMS1 homolog 2, mismatch repair system component (PMS2), and LS is the most common hereditary form of CRC (3-5).

Numerous studies have been published since the first case of hereditary CRC was reported in 1861 (6). Numerous microRNAs (miRNAs) and genes involved in the tumorigenesis of LS have been identified, such as miR-622, miR-1238 (3), insulin like growth factor 2 (7), phosphatase and tensin homolog (PTEN) (8), and PMS2 (4). In addition, certain cellular signaling pathways involved in the tumorigenesis of LS have been identified, including the AKT/mammalian target of rapamycin (mTOR) signaling pathways (8), axon guidance (9), and DNA repair pathways, such as the p53 pathway (10). In LS, somatic mutations of PTEN, which results in the upregulation of the AKT pathway, have been found and intervention against AKT or towards downstream targets, such as mTOR, resulted in a decreased incidence of LS, suggesting that AKT may be an effective approach for the prevention of HNPCC 
in patients $(8,9)$. However, the molecular mechanisms of LS remain to be elucidated.

In the study performed by Balaguer et al (3), hsa-miR-622, hsa-miR-1238 and hsa-miR-192 were identified as differentially expressed miRNAs (DEMs) in LS, compared with the sporadic microsatellite instability. However, the signatures of the identified target genes (TGs) of DEMs were not analyzed. In order to study the regulatory mechanisms of LS, the microarray data deposited by Balaguer et al were downloaded to identify key DEMs and their TGs. In addition, functional and pathway enrichment analyses were performed for TGs.

\section{Materials and methods}

miRNA microarray data. miRNA expression microarray data of GSE30454 (3) was downloaded from the Gene Expression Omnibus (GEO; http://www.ncbi.nlm.nih.gov/geo/), based on platform GPL8179 (Illumina Human v2 MicroRNA expression beadchip; Illumina Inc., San Diego, CA, USA). A total of 20 normal colonic tissue samples (N-C group) and $13 \mathrm{LS}$ tumor samples, consisting of 4 with a germline mutation in $M L H 1,5$ with a germline mutation in $M S H 2,3$ with a germline mutation in MSH6 and 1 with EpCam deletion (LS group) were selected (3). The raw data and the probe annotation files were downloaded for further analysis.

Data preprocessing and identification of LS-associated $D E M s$. Firstly, probe sets were mapped to the corresponding miRNAs. If there were multiple probe sets that corresponded to the same miRNA, the expression values of those probe sets were averaged. Then, the $t$-test method in the Linear Models for Microarray Data package of R (11) (limma version 3.22.7; www.bioconductor.org/packages/3.0/bioc/html/limma.html) was used to identify the DEMs between the $\mathrm{N}-\mathrm{C}$ and LS groups. Next, the $t$-test P-value was adjusted to the false discovery rate (FDR) by the Benjamini-Hochberg procedure (12). The cut-off criteria for DEMs were $\log _{2}$ fold change (FC) $\mid>1$ and FDR $<0.01$. Finally, the LS-associated DEMs were screened using the Human microRNA Disease Database (http://cmbi.bjmu.edu.cn/hmdd), which is a collection of experimentally supported human miRNA and disease associations (13).

Predication of TGs. From the standpoint of high confidence, the TGs of the LS-associated DEMs were predicted using 5 databases, as follows: miRanda (14); MirTarget2 (15); PicTar (16); PITA (17); and TargetScan (18). The intersections of the 5 databases were regarded as the final predicted TGs.

Functional and pathway enrichment analysis. Gene ontology (GO) analysis (http://www.geneontology.org/) is a functional method for the analysis of large-scale transcriptomic or genomic data (19). The Kyoto Encyclopedia of Genes and Genomes (KEGG) pathway database (http://www.genome.jp/kegg/pathway.html) contains information on the mechanism of molecules or genes (20). In order to investigate the biofunction of TGs in tumor progression, the Database for Annotation, Visualization and Integrated Discovery (DAVID; https://david.ncifcrf.gov/), a high-throughput and integrated data-mining environment (21), was used to perform the GO functional and KEGG pathway enrichment analyses for the TGs, based on the hypergeometric distribution. $\mathrm{P}<0.01$ was selected as the threshold.

Identification of transcription factors (TFs), tumor-associated genes (TAGs) and tumor suppressor genes (TSGs) among TGs. The TRANSFAC database (http://www.gene-regulation.com/pub/databases.html) is a database of eukaryotic transcription-regulating DNA sequence elements and the TFs binding to and acting through these elements (22). In order to determine whether the TGs had transcription regulation function, the TRANSFAC database was used to identify the TFs.

The Tumor Associated Gene (TAG) database (http://www.binfo.ncku.edu.tw/TAG/) is a semi-automatic information retrieving engine that was built to collect specific information on TGs from various resources (23). Additionally, Tumor Suppressor Gene (TSGene) database (http://bioinfo.mc.vanderbilt.edu/TSGene/) provides not only a comprehensive resource of TSGs for studies on cancer and to further experimental design, but also a comprehensive TSG catalog biology-based analyses of advanced systems (24). In order to identify the functions of the TGs that may function during genesis of LS, the TSGene and TAG databases were used to perform the identification of TSGs and TAGs among the TGs, respectively.

Construction of regulatory network. DEMs with $>100$ TGs were considered as key DEMs. According to the regulatory associations between key DEMs and TGs, the regulatory network containing key DEMs and their TGs was constructed and visualized by Cytoscape 2.8.2 software (25).

Statistical analysis. The t-test method in the Linear Models for Microarray Data package of R (limma, version 3.22.7) was used for the DEM selection. The P-value is adjusted as the FDR, and the selection criteria were $\log 2$ fold change (FC) $\mid>1$ and FDR $<0.01$. DAVID, based on the hypergeometric distribution, was used to perform GO and KEGG pathway enrichment analyses. $\mathrm{P}<0.01$ was considered to indicate a statistically significant difference.

\section{Results}

DEM identification and TG predication. A total of 159 DEMs were identified between the N-C and LS groups. There were 105 upregulated DEMs and 54 downregulated DEMs, of which only 13 DEMs were predicted to be associated with LS. Furthermore, 3 key DEMs were identified, including 2 upregulated miRNAs (hsa-miR-137 and hsa-miR-590-3p) and 1 downregulated miRNA (hsa-miR-520e). A total of 159 TGs for hsa-miR-137, including NCK adaptor protein 1 (NCK1), AKT serine/threonine kinase 2 (AKT2), Krüppel-like factor $(K L F)$ 4, metal-responsive transcription factor 1 (MTF1) and zinc finger protein $(Z N F)$ 217, 273 TGs for hsa-miR-590-3p, including $N C K 1$, hypoxia-inducible factor $1 \alpha(H I F 1 A), K L F 13$, nuclear factor I B (NFIB) and ZNF800 and 132 TGs for hsa-miR-520e, such as KLF13, NFIB, MTF1, ZNF800 and rapamycin-insensitive companion of mTOR (RICTOR), were identified. 


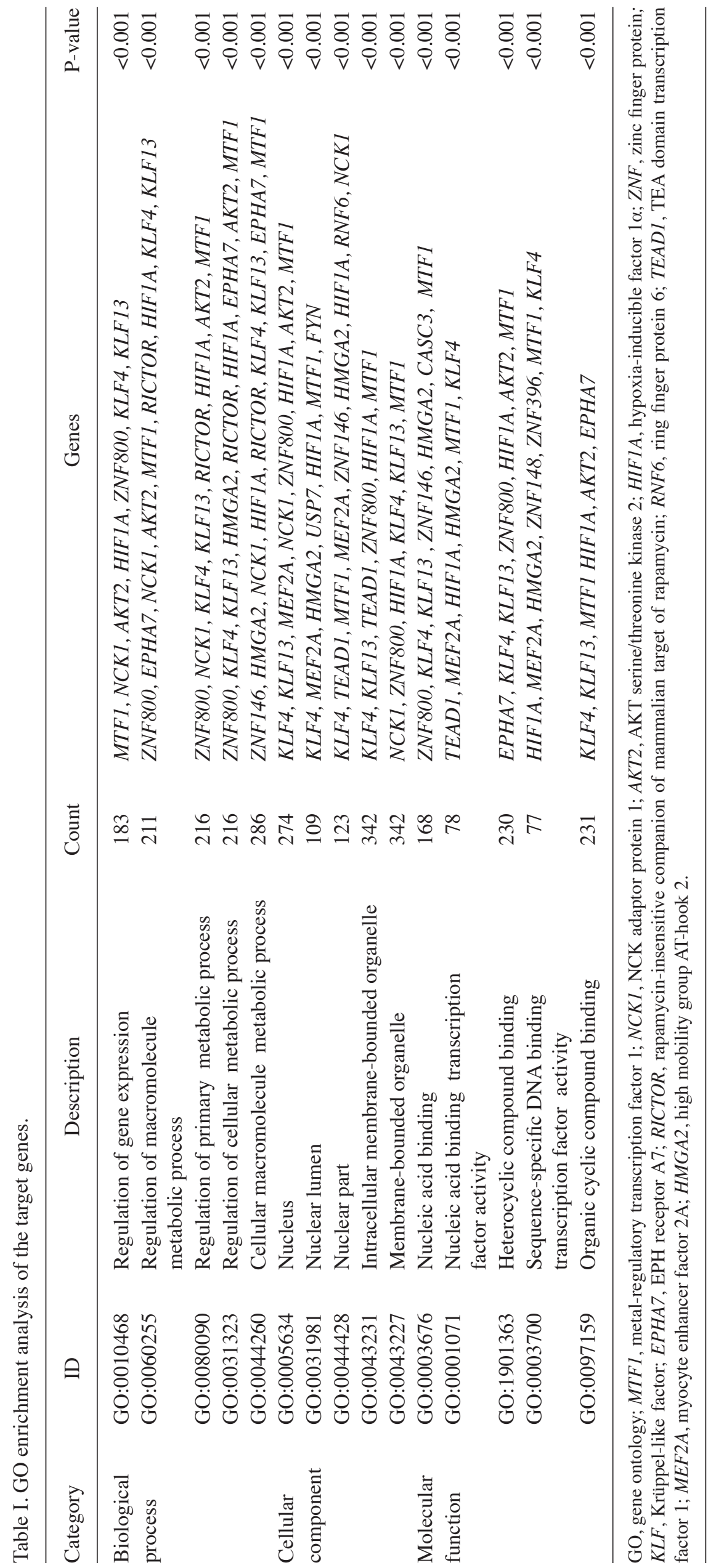


Table II. Kyoto Encyclopedia of Genes and Genomes pathway enrichment of the target genes.

\begin{tabular}{lcccr}
\hline ID & Description & Count & Genes & P-value \\
\hline 4150 & mTOR signaling pathway & 6 & CAB39, RICTOR, HIF 1A, AKT2, RPS6KA3, ULK1 & 0.001 \\
3013 & RNA transport & 9 & PAIP1, EIF3E, EIF4A2, EIF5, PABPC1, CASC3, EIF3J, NXT2, RANBP2 & 0.008 \\
4360 & Axon guidance & 8 & NCK1, FYN, EPHA7, SEMA3C, SEMA3E, PAK7, PPP3R1, CFL2 & 0.010 \\
\hline
\end{tabular}

mTOR, mammalian target of rapamycin; CAB39, calcium binding protein 39; RICTOR, rapamycin-insensitive companion of mammalian target of rapamycin; HIF1A, hypoxia-inducible factor $1 \alpha$; AKT2, AKT serine/threonine kinase 2; RPS6KA3, ribosomal protein S6 kinase A3; $U L K 1$, unc-51 like autophagy activating kinase 1; PAIP1, poly(A) binding protein interacting protein 1; EIF, eukaryotic translation initiation factor; $P A B P C 1$, poly(A) binding protein cytoplasmic 1; CASC3, cancer susceptibility candidate 3; NXT2, nuclear transport factor 2 like export factor 2; RANBP2, RAN binding protein 2; NCK1, NCK adaptor protein 1; FYN, FYN proto-oncogene, Src family tyrosine kinase; EPHA7, EPH receptor A7; SEMA, semaphorin; PAK7, p21 (RAC1) activated kinase 7; PPP3R1, protein phosphatase 3 regulatory subunit B, $\alpha$; $C F L 2$, cofilin 2.

Functional and pathway enrichment analysis. Through the $\mathrm{GO}$ functional enrichment analysis of biological processes, certain TGs, including NCK1, HIF1A, MTF1, KLF13, KLF4 and $A K T 2$, were found to be involved in the regulation of gene expression $(\mathrm{P}<0.001)$. Certain TGs, including NCK1, MTF1, KLF4, KLF13, HIF1A, AKT2 and RICTOR, were involved in the regulation of macromolecule metabolic processes $(\mathrm{P}<0.001)$ and the regulation of primary metabolic processes $(\mathrm{P}<0.001)$ (Table I). Through the GO functional enrichment analysis of cellular components, certain TGs, including $M T F 1$, $K L F 13, N C K 1, H I F 1 A$ and AKT2, were found to be enriched in the nucleus $(\mathrm{P}<0.001)$. Certain TGs, including HIFlA, $M T F 1, K L F 4, K L F 13$ and ZNF800, were enriched in intracellular membrane-bound organelles $(\mathrm{P}<0.001)$; certain TGs, including MTF1, KLF13, NCK1, HIF1A and ZNF800, were enriched in membrane-bound organelles $(\mathrm{P}<0.001)$ (Table I). Through the GO functional enrichment analysis of molecular function, certain TGs, such as MTF1, KLF4, KLF13, HIF1A, ZNF800, and cancer susceptibility candidate 3 (CASC3), were involved in nucleic acid binding $(\mathrm{P}<0.001)$. In addition, certain TGs, such as $M T F 1, K L F 4$, TEA domain transcription factor 1 (TEAD1), zinc finger protein 148, and HIF1A, were involved in nucleic acid binding TF activity $(\mathrm{P}<0.001)$. Certain TGs, such as MTF1, KLF4, KLF13, HIF1A, AKT2 and ZNF800, were involved in heterocyclic compound binding $(\mathrm{P}<0.001)$ (Table I).

According to the KEGG pathway enrichment analysis, TGs were significantly enriched in 3 pathways: mTOR signaling pathway, including RICTOR, HIF1A and AKT2 ( $\mathrm{P}=0.001)$; RNA transport, including eukaryotic translation initiation factor $(E I F) 3$ subunit E, CASC3 and EIF3J ( $\mathrm{P}=0.008)$; and axon guidance, including $N C K 1$, FYN proto-oncogene, $\mathrm{Src}$ family tyrosine kinase $(F Y N)$ and EPH receptor A7 (EPHA7) $(\mathrm{P}=0.010)$ (Table II).

Identification of TFs, TSGs and TAGs. A total of 12 TGs, consisting of TEAD1, ZNF146, high mobility group AT-hook 2 (HMGA2), FYN, v-crk avian sarcoma virus CT10 oncogene homolog-like, receptor-like tyrosine kinase, ZNF217, KIT proto-oncogene receptor tyrosine kinase, $A K T 2$, chromosome segregation 1 like, interferon regulatory factor 2 and B-cell CLL/lymphoma 6 (BCL6), were identified as oncogenes, according to the TSGene database and TAG database. Among these identified oncogenes, 4 genes, consisting of TEAD1, ZNF146, HMGA2 and BCL6, were identified as TFs based on the TRANSFAC database.

Regulatory network construction. The visualized regulatory network of the key DEMs and the TGs of these DEMs showed that hsa-miR-520e and hsa-miR-137 had 4 common TGs, consisting of SNF related kinase (SNRK), MTF1, round spermatid basic protein $1(R S B N I)$ and YTH N6-methyladenosine RNA binding protein 3 (YTHDF3), hsa-miR-590-3p and hsa-miR-137 had 14 common TGs, including NCK1, EPHA7, stress-associated endoplasmic reticulum protein $1(S E R P I)$ and myocyte enhancer factor 2A (MEF2A), and hsa-miR-590-3p and hsa-miR-520e had 12 common TGs, including KLF13, twinfilin actin binding protein 1 (TWF1), NFIB and ZNF800 (Fig. 1).

\section{Discussion}

In the current study, 2 upregulated miRNAs, consisting of hsa-miR-137 and hsa-miR-590-3p, and 1 downregulated miRNA, hsa-miR-520e, were screened as key DEMs. Hsa-miR-520e and hsa-miR-137 had 4 common TGs, consisting of SNRK, MTF1, RSBN1 and YTHDF3. Through functional enrichment analysis, $M T F 1$ was identified as involved in the regulation of gene expression and macromolecule metabolic processes, intracellular membrane-bound organelles, and sequence-specific DNA binding TF activity. As previously reported, MTF1 binds specifically to heavy metal-responsive DNA sequence elements in the enhancer/promoter region of metallothionein (MT) genes to regulate their expression (26,27). MT genes, such as $M T 1 X$ and $M T 2 A$, encode small cysteine-rich proteins that scavenge heavy metals, such as $\mathrm{Zn}$ (II), Cd (II) and Cu (I), and reactive oxygen species $(28,29)$. Arriaga et al (30) found that the expression of 5 isoforms of MTs, consisting of MT1G, MT1E, MT1F, MT1H and MT1M, were lost during the transition between normal colorectal mucosa and CRC, and the expression of MTs was associated with a shorter survival time in CRC patients (30). In addition, the MTIX gene showed a high sensitivity for the identification of patients with LS or CRC with sporadic defective in MMR (31). This suggests that hsa-miR-520e and hsa-miR-137 may have crucial roles in LS by regulating TGs. 


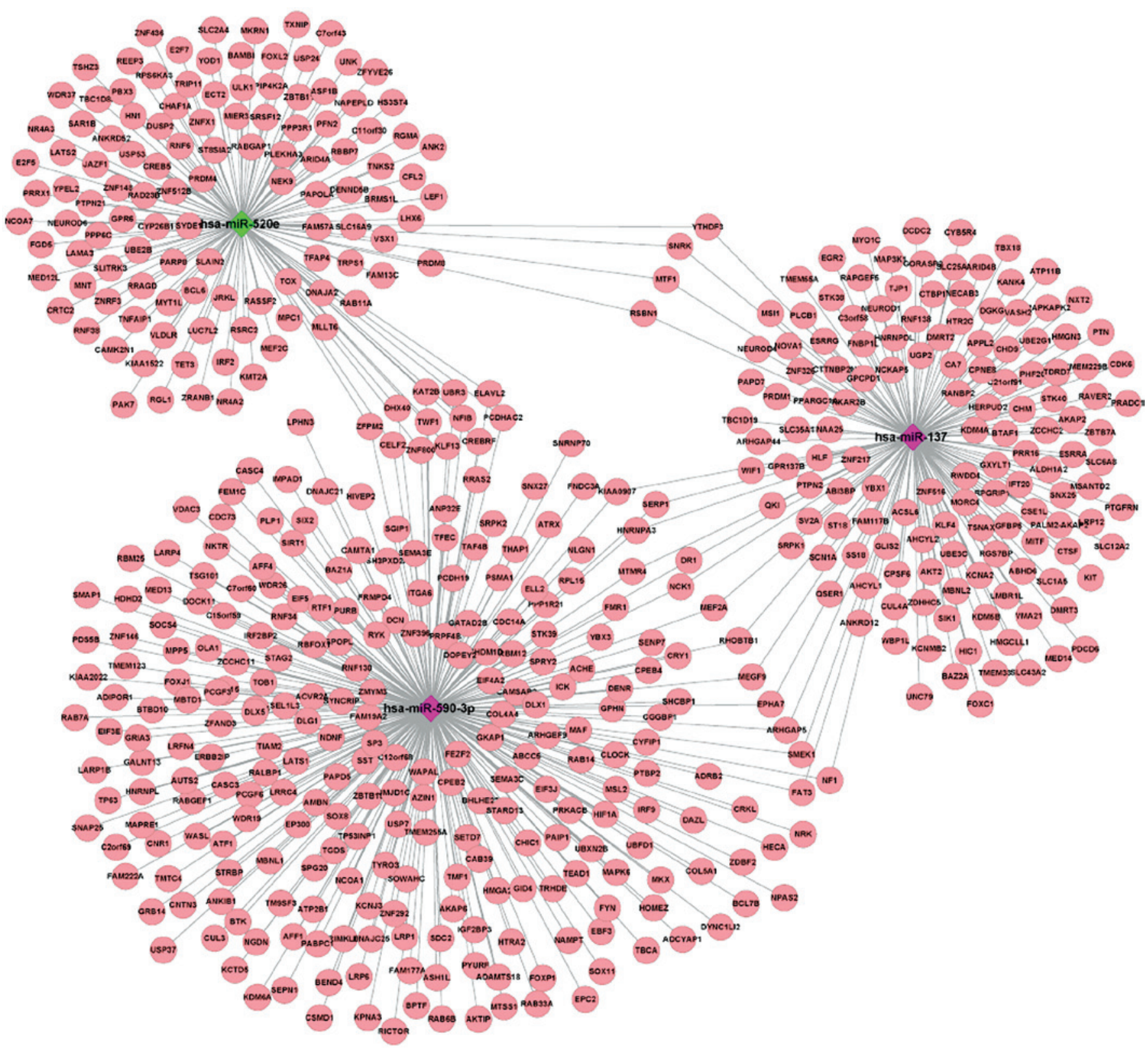

Figure 1. Regulatory network of the 3 key differentially expressed miRNAs and their TGs. Nodes: Ellipses indicate the TGs; diamonds indicate the upregulated miRNAs; triangles indicate the downregulated miRNAs; intermolecular interactions between miRNAs and their TGs are indicated by links. TGs, target genes; miRNA, microRNA.

In the present study, hsa-miR-590-3p and hsa-miR-520e had 12 common TGs, including KLF13, TWF1, NFIB and ZNF800, while KLF4 was the TG of hsa-miR-137. Using functional enrichment analysis, KLF4 and KLF13 were identified as involved in the regulation of gene expression, heterocyclic compound binding, and regulation of the cellular metabolic process. LOR-253, a compound that stimulates KLF4 through the inhibition of the human $M T F 1$, is currently used in an early stage of colon cancer (32). The KLF proteins are zinc finger-containing TFs that exert important functions in regulating diverse biological processes, such as growth and cell proliferation (33). KLF4, formerly termed $G K L F$, encodes a TF associated with tumor suppression and oncogenesis (34). In LS, KLF4 and KLF5 were expressed and predominantly localized to the epithelial cells of the tumors (35). Overexpression of KLF4 in the colon cancer cell lines resulted in reduced tumorigenesis (36). Additionally, a previous study reported that the loss of heterozygosity in the KLF6 locus in patients with sporadic CRC and LS was $0-35 \%$ and $0 \%$, respectively (37). These data suggested that loss of heterozygosity of the KLF6 locus was rarely involved in the carcinogenesis of CRC in patients with LS (38). This revealed that hsa-miR-590-3p and hsa-miR-520e, in addition to hsa-miR-137, may have important roles in the cellular metabolic process and proliferation in LS by targeting their TGs.

Additionally, the common TGs, including NCK1, EPHA7, SERP1 and MEF2A, were simultaneously regulated by hsa-miR-590-3p and hsa-miR-137. The enrichment analysis showed that $N C K 1$ was significantly enriched in the axon guidance pathway. As previously reported, AKT executes double roles in protecting motoneuronal survival and promoting nerve regeneration in vivo, and the dominant overexpression 
of $A K T$ in adult hypoglossal neurons showed accelerated axonal regeneration (39). However, the phosphorylation of $A K T$ was partially reduced in $N C K 1$-deficient B cells, and it was almost completely absent in $N C K 1-N C K 2$-deficient $\mathrm{B}$ cells. This revealed that the activation of the phosphoinositide 3-kinase (PI3K)/AKT pathway was restrained in the NCK1-NCK2-knockout B cells (40). Furthermore, ectopic expression of hsa-miR-137 in CRC cells inhibited the phosphorylation of mitogen-activated protein kinase (MAPK) and AKT, which reduced the invasiveness of CRC cells by inhibiting signaling via the P13K/AKT and MAPK pathways (41). Hsa-miR-590-3p was also found to activate the PI3K/AKT signaling pathway by downregulating PTEN, a TSG-activated $\mathrm{P} 13 \mathrm{~K}$ pathway, and then provide strong growth and survival signals to tumor cells (42). Thus, these results suggested that hsa-miR-590-3p and hsa-miR-137 may play important roles in LS by regulating their target genes through the axon guidance pathway.

The functional and pathway enrichment analyses showed that certain TGs of the key DEMs, including HIF 1A, AKT2 and RICTOR, participated in the mTOR signaling pathway. mTOR is a member of the PI3K/AKT/mTOR pathway, whose activation stimulates protein and lipid biosynthesis and it is constitutively activated in LS (43). The mTOR signaling pathway senses and integrates a variety of environmental cues to regulate numerous major cellular processes $(44,45)$. As previously shown, the mTOR complex 2 protein RICTOR was highly overexpressed in CRC tissues, and the inhibition of RICTOR resulted in growth inhibition and induced apoptosis in CRCs $(46,47)$. Additionally, AKT2, a component of the PI3K/AKT/mTOR pathway, is proposed as an oncogene for pancreatic cancer that always occurs in the context of LS (48), and the overexpression of the $A K T 2$ proto-oncogene is an early event during sporadic colon cancer $(47,49)$. In addition, mTOR directly stimulated HIFlA, downstream of PI3K/AKT/mTOR, and indirectly causes other metabolic changes by activating $\operatorname{HIF} 1 A(50,51)$. The subsequent HIF1-dependent metabolic changes are a major determinant of the glycolytic phenotype, including the Warburg effect $(50,51)$. The Warburg effect in CRC results in the accumulation of a glycolytic metabolite, pyruvate, which provides the CRC cells with an environment that is a competitive advantage for invasion (52). Overexpression of HIF1A has also been reported to be significantly associated with shorter CRC-specific survival and overall survival times (53). In the present study, AKT2 was a TG of hsa-miR-137, while RICTOR and HIFlA were TGs of hsa-miR-590-3p. All these genes were enriched in the regulation of the metabolic process. Therefore, it may be hypothesized that the important roles of hsa-miR-137 and hsa-miR-590-3p in regulating metabolic process occur by regulating the expression of their TGs in LS.

In the present study, 3 key DEMs, consisting of hsa-miR-520e, hsa-miR-590-3p and hsa-miR-137 were identified. Hsa-miR-590-3p and hsa-miR-520e had 12 common TGs, including KLF13. Hsa-miR-590-3p and hsa-miR-137 had 14 common TGs, including NCK1. Hsa-miR-520e and hsa-miR-137 had 4 common TGs, including MTF1. Functional and pathway enrichment analysis showed that these TGs were significantly enriched in important functions, such as the regulation of metabolic processes, including regulation of macromolecule metabolic process, regulation of primary metabolic process, regulation of cellular metabolic process and cellular macromolecule metabolic process, mTOR signaling, and the axon guidance pathway, which were crucial for the growth and regulation of LS cancer cells. The present study showed the potential crucial role of hsa-miR-520e, hsa-miR-590-3p and hsa-miR-137 in LS.

In conclusion, the present study identified that hsa-miR-137, hsa-miR-520e and hsa-miR-590-3p are key DEMs. Hsa-miR-520e and hsa-miR-137 had 4 common TGs, consisting of SNRK, MTF1, RSBN1 and YTHDF3. Hsa-miR-590-3p and hsa-miR-137 had 14 common TGs, including NCK1, EPHA7 and SERP1. Hsa-miR-590-3p and hsa-miR-520e had 12 common TGs, including KLF13, TWF1 and NFIB. These regulatory interactions may provide biomarkers for LS detection and prognosis.

\section{References}

1. Siegel R, DeSantis C and Jemal A: Colorectal cancer statistics, 2014. CA Cancer J Clin 64: 104-117, 2014.

2. Jemal A, Siegel R, Ward E, Hao Y, Xu J and Thun MJ: Cancer statistics, 2009. CA Cancer J Clin 59: 225-249, 2009.

3. Balaguer F, Moreira L, Lozano JJ, Link A, Ramirez G, Shen Y, Cuatrecasas M, Arnold M, Meltzer SJ, Syngal S, et al: Colorectal cancers with microsatellite instability display unique miRNA profiles. Clin Cancer Res 17: 6239-6249, 2011.

4. Lynch HT, Lynch PM, Lanspa SJ, Snyder CL, Lynch JF and Boland CR: Review of the Lynch syndrome: History, molecular genetics, screening, differential diagnosis and medicolegal ramifications. Clin Genet 76: 1-18, 2009.

5. Lynch HT, Boland CR, Gong G, Shaw TG, Lynch PM, Fodde R, Lynch JF and de la Chapelle A: Phenotypic and genotypic heterogeneity in the Lynch syndrome: Diagnostic, surveillance and management implications. Eur J Hum Genet 14: 390-402, 2006.

6. Luschka H: Ueber polypöse Vegetationen der gesammten Dickdarmschleimhaut. Virchow's Arch f path Anat 20: 133-142, 1861.

7. Jass JR: Heredity and DNA methylation in colorectal cancer. Gut 56: 154-155, 2007.

8. Qian CN, Furge KA, Knol J, Huang D, Chen J, Dykema KJ, Kort EJ, Massie A, Khoo SK, Vanden Beldt K, et al: Activation of the PI3K/AKT pathway induces urothelial carcinoma of the renal pelvis: Identification in human tumors and confirmation in animal models. Cancer Res 69: 8256-8264, 2009.

9. van Engeland M, Derks S, Smits KM, Meijer GA and Herman JG: Colorectal cancer epigenetics: Complex simplicity. J Clin Oncol 29: 1382-1391, 2011.

10. Murphy ME: Polymorphic variants in the p53 pathway. Cell Death Differ 13: 916-920, 2006.

11. Wettenhall JM and Smyth GK: limmaGUI: A graphical user interface for linear modeling of microarray data. Bioinformatics 20: 3705-3706, 2004.

12. Benjamini Y and Hochberg Y: Controlling the false discovery rate: A practical and powerful approach to multiple testing. J Roy Stat Soc B 57: 289-300, 1995.

13. Leidinger P, Keller A, Borries A, Reichrath J, Rass K, Jager SU, Lenhof HP and Meese E: High-throughput miRNA profiling of human melanoma blood samples. BMC Cancer 10: 262, 2010.

14. Dweep H, Sticht C, Pandey P and Gretz N: miRWalk-database: Prediction of possible miRNA binding sites by 'walking' the genes of three genomes. J Biomed Inform 44: 839-847, 2011.

15. Wang $X$ and El Naqa IM: Prediction of both conserved and nonconserved microRNA targets in animals. Bioinformatics 24: 325-332, 2008.

16. Krek A, Grün D, Poy MN, Wolf R, Rosenberg L, Epstein EJ, MacMenamin P, da Piedade I, Gunsalus KC, Stoffel M and Rajewsky N: Combinatorial microRNA target predictions. Nat Genet 37: 495-500, 2005.

17. Betel D, Koppal A, Agius P, Sander C and Leslie C: Comprehensive modeling of microRNA targets predicts functional non-conserved and non-canonical sites. Genome Biol 11: R90, 2010.

18. Lewis BP, Shih IH, Jones-Rhoades MW, Bartel DP and Burge CB: Prediction of mammalian microRNA targets. Cell 115: 787-798, 2003. 
19. Hulsegge I, Kommadath A and Smits MA: Globaltest and GOEAST: Two different approaches for Gene ontology analysis. BMC Proc 3 (Suppl 4): S10, 2009.

20. Kanehisa M and Goto S: KEGG: Kyoto encyclopedia of genes and genomes. Nucleic Acids Res 28: 27-30, 2000.

21. Huang da W, Sherman BT and Lempicki RA: Systematic and integrative analysis of large gene lists using DAVID bioinformatics resources. Nat Protoc 4: 44-57, 2009.

22. Wingender E, Dietze P, Karas H and Knüppel R: TRANSFAC: A database on transcription factors and their DNA binding sites. Nucleic Acids Res 24: 238-241, 1996

23. Chen JS, Hung WS, Chan HH, Tsai SJ and Sun HS: In silico identification of oncogenic potential of fyn-related kinase in hepatocellular carcinoma. Bioinformatics 29: 420-427, 2013.

24. Zhao M, Sun J and Zhao Z: TSGene: A web resource for tumor suppressor genes. Nucleic Acids Res 41 (Database issue) D970-D976, 2013.

25. Shannon P, Markiel A, Ozier O, Baliga NS, Wang JT, Ramage D, Amin N, Schwikowski B and Ideker T: Cytoscape: A software environment for integrated models of biomolecular interaction networks. Genome Res 13: 2498-2504, 2003.

26. Boudreault F, Pinilla M, Kho A, Baron R and Tschumperlin D: Metallothionein is a stretch-induced gene that confers protection during mechanical ventilation. Technology 1: 3, 2011.

27. Günther V, Lindert $U$ and Schaffner W: The taste of heavy metals: Gene regulation by MTF-1. Biochim Biophys Acta 1823 1416-1425, 2012

28. Saini N, Georgiev O and Schaffner W: The parkin mutant phenotype in the fly is largely rescued by metal-responsive transcription factor (MTF-1). Mol Cell Biol 31: 2151-2161, 2011.

29. Günther V, Davis AM, Georgiev O and Schaffner W: A conserved cysteine cluster, essential for transcriptional activity, mediates homodimerization of human metal-responsive transcription factor-1 (MTF-1). Biochim Biophys Acta 1823: 476-483, 2012.

30. Arriaga JM, Levy EM, Bravo AI, Bayo SM, Amat M, Aris M, Hannois A, Bruno L, Roberti MP, Loria FS, et al: Metallothionein expression in colorectal cancer: Relevance of different isoforms for tumor progression and patient survival. Hum Pathol 43 : 197-208, 2012

31. Morandi L, de Biase D, Visani M, Monzoni A, Tosi A, Brulatti M, Turchetti D, Baccarini P, Tallini G and Pession A: T ([20]) repeat in the 3'-untranslated region of the MT1X gene: A marker with high sensitivity and specificity to detect microsatellite instability in colorectal cancer. Int J Colorectal Dis 27: 647-656, 2012

32. Constant S, Huang S, Wiszniewski L and Mas C (eds): Colon cancer: Current treatments and preclinical models for the discovery and development of new therapies. In: Drug Discovery. El-Shemy HA (ed). InTech, Rijeka, pp433-458, 2013.

33. Ghaleb AM and Yang VW: The pathobiology of Krüppel-like factors in colorectal cancer. Current Colorectal Cancer Rep 4 59-64, 2008

34. Rowland BD, Bernards R and Peeper DS: The KLF4 tumour suppressor is a transcriptional repressor of p53 that acts as a context-dependent oncogene. Nat Cell Biol 7: 1074-1082, 2005.

35. Shao J, Yang VW and Sheng H: Prostaglandin E2 and Krüppel-like transcription factors synergistically induce the expression of decay-accelerating factor in intestinal epithelial cells. Immunology 125: 397-407, 2008.

36. Dang DT, Chen X, Feng J, Torbenson M, Dang LH and Yang VW: Overexpression of Krüppel-like factor 4 in the human colon cancer cell line RKO leads to reduced tumorigenecity. Oncogene 22: 3424-3430, 2003.
37. Saunders IW, Ross J, Macrae F, Young GP, Blanco I, Brohede J, Brown G, Brookes D, Lockett T, Molloy PL, et al: Evidence of linkage to chromosomes 10p15.3-p15.1, 14q24.3-q31.1 and 9q33.3-q34.3 in non-syndromic colorectal cancer families. Eur J Hum Genet 20: 91-96, 2012.

38. Miyaki M, Yamaguchi T, Iijima T, Funata N and Mori T: Difference in the role of loss of heterozygosity at 10p15 (KLF6 locus) in colorectal carcinogenesis between sporadic and familial adenomatous polyposis and hereditary nonpolyposis colorectal cancer patients. Oncology 71: 131-135, 2006.

39. Namikawa K, Honma M, Abe K, Takeda M, Mansur K, Obata T, Miwa A, Okado $\mathrm{H}$ and Kiyama H: Akt/protein kinase B prevents injury-induced motoneuron death and accelerates axonal regeneration. J Neurosci 20: 2875-2886, 2000.

40. Leu CM: Nck, a missing adaptor between the B-cell receptor complex and the BCAP/PI3K/Akt pathway. Cell Mol Immunol 11: 120-122, 2014

41. Liang L, Li X, Zhang X, Lv Z, He G, Zhao W, Ren X, Li Y, Bian X, Liao W, et al: MicroRNA-137, an HMGA1 target, suppresses colorectal cancer cell invasion and metastasis in mice by directly targeting FMNL2. Gastroenterology 144: 624 . e4-635.e4, 2013.

42. Yang H, Zheng W, Zhao W, Guan C and An J: Roles of miR-590-5p and miR-590-3p in the development of hepatocellular carcinoma. Nan Fang Yi Ke Da Xue Xue Bao 33: 804-811, 2013 (In Chinese).

43. Ekstrand AI, Jönsson M, Lindblom A, Borg A and Nilbert M: Frequent alterations of the PI3K/AKT/mTOR pathways in hereditary nonpolyposis colorectal cancer. Fam Cancer 9: 125-129, 2010.

44. Laplante M and Sabatini DM: mTOR signaling in growth control and disease. Cell 149: 274-293, 2012.

45. Wullschleger S, Loewith $\mathrm{R}$ and Hall MN: TOR signaling in growth and metabolism. Cell 124: 471-484, 2006.

46. Gulhati P, Cai Q, Li J, Liu J, Rychahou PG, Qiu S, Lee EY, Silva SR, Bowen KA, Gao T and Evers BM: Targeted inhibition of mammalian target of rapamycin signaling inhibits tumorigenesis of colorectal cancer. Clin Cancer Res 15: 7207-7216, 2009.

47. Johnson SM, Gulhati P, Rampy BA, Han Y, Rychahou PG, Doan HQ, Weiss HL and Evers BM: Novel expression patterns of PI3K/Akt/mTOR signaling pathway components in colorectal cancer. J Am Coll Surg 210: 767-776, 2010.

48. Schneider G and Schmid RM: Genetic alterations in pancreatic carcinoma. Mol Cancer 2: 15, 2003.

49. Roy HK, Olusola BF, Clemens DL, Karolski WJ, Ratashak A, Lynch HT and Smyrk TC: AKT proto-oncogene overexpression is an early event during sporadic colon carcinogenesis. Carcinogenesis 23: 201-205, 2002

50. Cairns RA, Harris IS and Mak TW: Regulation of cancer cell metabolism. Nat Rev Cancer 11: 85-95, 2011.

51. Mellinghoff IK and Sawyers CL: TORward AKTually useful mouse models. Nat Med 10: 579-580, 2004.

52. Bi X, Lin Q, Foo TW, Joshi S, You T, Shen HM, Ong CN, Cheah PY, Eu KW and Hew CL: Proteomic analysis of colorectal cancer reveals alterations in metabolic pathways: Mechanism of tumorigenesis. Mol Cell Proteomics 5: 1119-1130, 2006

53. Baba Y, Nosho K, Shima K, Irahara N, Chan AT, Meyerhardt JA, Chung DC, Giovannucci EL, Fuchs CS and Ogino S: HIF1A overexpression is associated with poor prognosis in a cohort of 731 colorectal cancers. Am J Pathol 176: 2292-2301, 2010. 